\title{
STUDIES ON NOVEL REARANGEMENT OF BENZOXAZOLETHIOL
}

\author{
"Samira Abou-Srie Swelam' and Sherifa Mostafa Abu-Bakr' \\ Department of Photochemistry ${ }^{i} \&$ Chemistry of Natural and Microbial Products ${ }^{2}$ National \\ Research Centre, Dokki, Cairo Egypt \\ samira_swellam@yahoo.com
}

\begin{abstract}
A new chemoselective approach to synthesize a series of benzoimidazole 2a-c, 3, 4a, b and benzoxazol-2-thiol derivatives 5a-c, 6, 7a,b. Benzoxazol-2-thiol derivatives under action of $\mathrm{N}$-nucleophiles, by using this methodology, two series of heterocyclic systems were synthesized. 7-Chloro-2-methyl/or aryl-benzo-[4,5]imidazo[1,2,4] thiadiazole derivatives 8,9 and 10a-c were prepared via cyclocondensation and condensation elimination reaction.

Key words: Benzoxazolthiol, chemoselective, benzoimidazol, N- nucleophiles
\end{abstract}

\section{Introduction}

A review of the literature revealed that compounds possessing benzoxazole moiety showed significant (in vitro) antibacterial activity especially against some enteric Gramnegative rods suchas Klebsiella pneumoniae, $P$ seudomoanas aerugiunasa and the yeast Candida albicans[1-8]. Therefore, we directed our research to elaborate chemoselective procedures for the synthesis of a variety of hetero cyclic compounds of biological interest.

\section{Result and Discussion}

As a result of our research we found that depending on reaction conditions, 5-substitutedbenzoxazl-2-thiol (1) reacted with different $\mathrm{N}$ - nucleophiles in two ways, namely, oxazole ring opening (Figure-1) and without one (Figure-3).

Indeed, in glacial acetic acid at refluxing temperature, reaction between benzoxazol-2thiol derivative 1 and different primary amines, hydrazine hydrate and substituted hydrazine hydrates took place with ring opening [9] (Figure-1).

The benzoimidazole derivatives were synthesized via stirring of 1 with primary amines, hydrazine hydrate and substituted phenyl hydrazine in presence of $n$-butanol at room temperature. 'H-NMR of these compounds shows signals at $\mathrm{d}$ (ppm ) 2.32, 2.31, 2.12, 2.19, 2.09, $2.12\left(\mathrm{~s}, 1 \mathrm{H}\right.$, exchangeable with $\left.\mathrm{D}_{2} \mathrm{O}, \mathrm{SH}\right)$ for $\mathbf{2 a - c ,} 3, \mathbf{4 a}, \mathbf{b}$ (cf.,exp). 


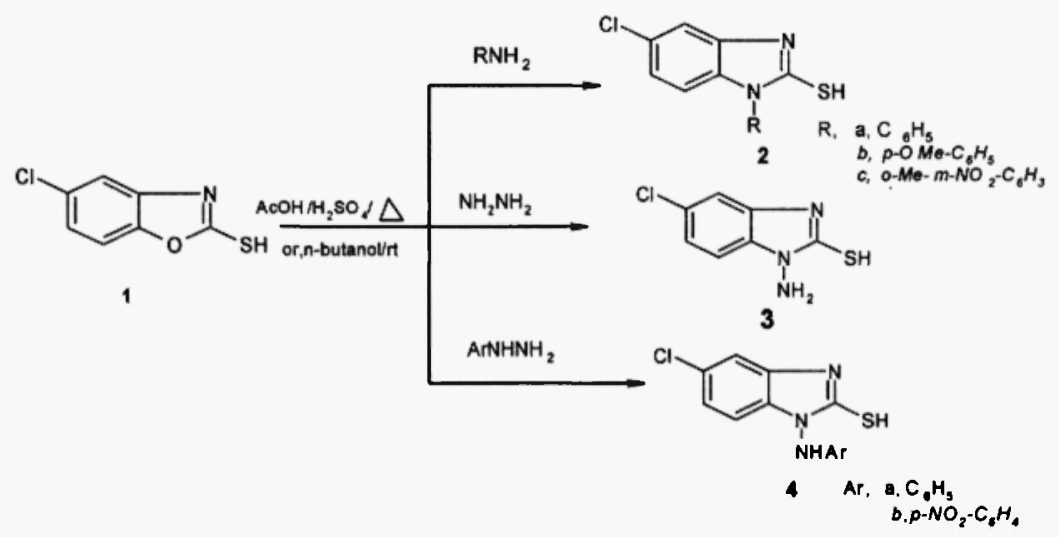

Figure-1

A mechanism that can be accounted for these products ( $2 \mathbf{a}-\mathbf{c}, \mathbf{3}, \mathbf{4 a}, \mathbf{b})$ may be analogous to acidic hydrolysis of benzoxazol-2-thiol derivative to the corresponding II \& III (Figure-2). Therefore, when the reaction carried out in aqueous acidic media, the reaction mechanism might take place through :

i) Formation of 2-(R-imino)-benzoxazol-2-thiol derivatives. ii) Nucleophilic attack of water on $\mathrm{C}_{2}$ of oxazol ring with simultaneous ring opening. iii) $\mathrm{E}_{2}$ Isomerization and subsequent cyclization of III to form $\mathrm{N}$-substitutedbenzoxazol-2-thiol derivatives [10] (Figure-2).

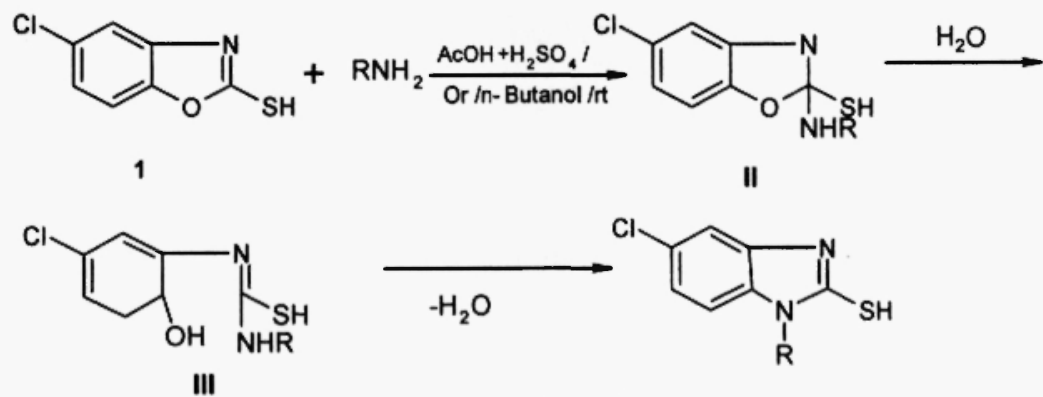

\section{Figure-2}

Starting from our assumption of two possible mechanisms of the rearrangements and varying reaction conditions from aqueous acidic media, acidic media ( $80 \%$ acetic acid and drops of sulfuric acid) and solvent with high boiling point such as n-butanol, we have benzoxazole derivatives 5a-c, 6 and 7a, b (Figure-3). 
5-Chlorobenzoxazole derivatives 5a-c, 6, 7a,b were synthesized when 1 stirred under refluxing temperature for $10 \mathrm{hrs}$ with primary amines, hydrazine hydrate and substituted phenyl hydrazine in presence of $n$-butanol. The release of hydrogen sulfides were detected through the reaction.

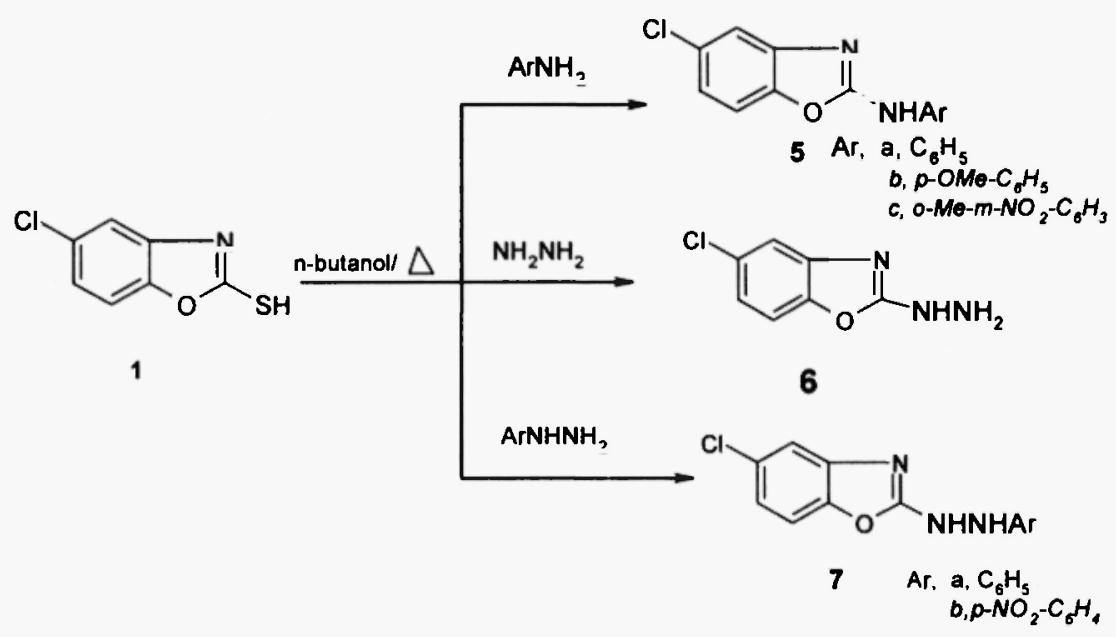

Figure-3

A possible mechanism of this feature might take place via : i) Nucleophilc attack of $\mathrm{NH}_{2}$ on $\mathrm{C}_{2}$ of benzoxazol ring . ii) Ring opening and $\mathrm{E} / \mathrm{Z}$ isomerization of the intermediate (IV,V ). iii) Subsequent liberation of hydrogen sulfide [11] (Figure -4).

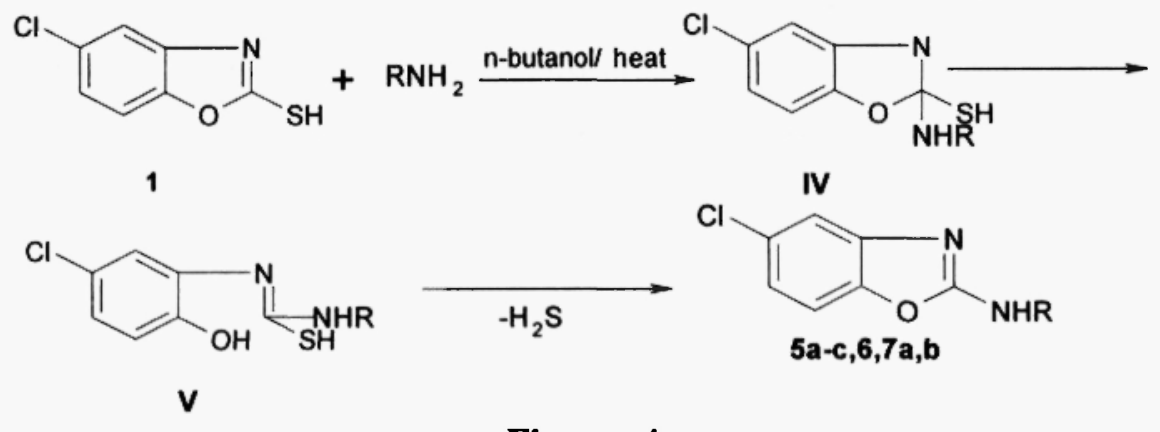

\section{Figure -4}

In continuation of our interest in the synthesis of fused heterocyclic compounds [12-15]. We reported here simple methods for synthesis of 7-chloro-2-methyl/or aryl- benzo$[4,5]$ imidazo[ $1,2,4]$ thiadiazole 8 and 10 a-c through cyclocondensation reactions upon treating with triethylorothoacetate, and aromatic aldehydes namely (benzaldehyde, anisaldehyde and N,N- dimethylaminobenzaldehyde) [12-15,17] (Figure-5). 
Similarly[17], reaction of $\mathbf{3}$ with ethylchloroformate in presence of pyridine afforded 9 (Figure-5). The mass spectra showed expected molecular ion peak at 225.59 (53\%). IR spectra exhibit absorption bands at $\mathrm{cm}^{-i} 3120(\mathrm{NH})$ and 1670 (cyclic amide) and the structure of 9 was confirmed from its ' H-NMR ( exp. ).

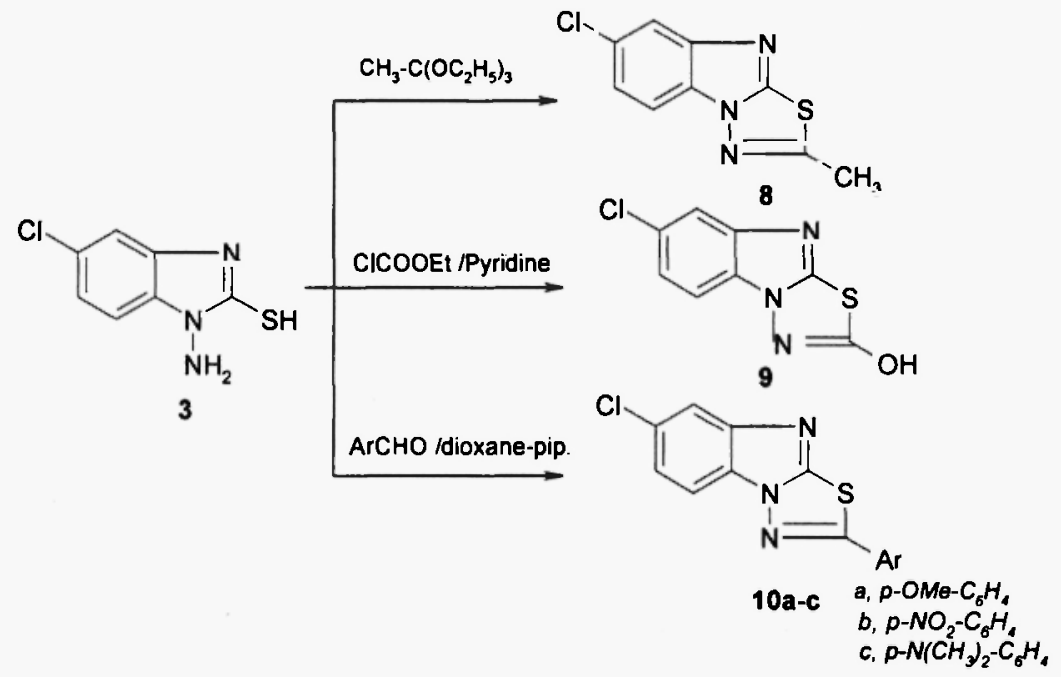

\section{Experimental}

Figure-5

Melting points are uncorrected, the purity and the reaction controlled time were detected by TLC, Microanalyses were performed by micro analytical Unit, NRC. All compounds gave satisfactory values for $\mathrm{C}, \mathrm{H}, \mathrm{Cl}, \mathrm{N}$ and $\mathrm{S}$ within range of \pm 0.04 . IR spectra $(\mathrm{KBr})$ were recorded on Perkin Elmer 580 spectrophotometer. ${ }^{~} \mathrm{H}$-NMR were carried on JNM,FTNMR-EX270, run ' $\mathrm{H}-\mathrm{NMR} 270 \mathrm{MHz}$, in DMSO-d 6 using TMS as internal standard and chemical shifts are expressed in d ppm. Mass spectra were recorded on Varian Mat 112 spectrometer.

5-Chloro-benzoxazol-2-thiol 1[17].

5-Chloro-1-aryl-1H-benzoimidazole-2-thiol 2a-c

1-Amino-5-chloro-1H-benzoimidazole-2-thiol 3

1-Arylamino-5-chloro-1 H-benzoimidazole-2-thiol4a,b

\section{Method 1}

An equimolar amounts $(0.37 \mathrm{~g}, 2 \mathrm{mmol})$ of 1 and appropriate aromatic amine (aniline, $p$ anisidine and $m$-nitro-o-toluedine), hydrazin hydrate. phenylhydrazine or $p$-nitrophenyl 
hydrazine was heated in $30 \mathrm{ml}$ acetic acid and one drop of sulfuric acid for $9 \mathrm{hrs}$. The reaction mixture was cooled, poured into crushed ice and the solid so formed was collected by filtration and crystallized from appropriate solvent .

\section{Method 2}

An equimolar amounts $(0.37 \mathrm{~g}, 2 \mathrm{mmol})$ of $\quad 1$ and appropriate aromatic amine (aniline, $\quad p$ anisidine and $m$-nitro-o-toluedine), hydrazine hydrate, phenyl hydrazine or $p$-nitrophenyl hydrazine was stirred in $30 \mathrm{ml} \mathrm{n}$-butanol at room temperature for $10 \mathrm{hrs}$. The reaction mixture was left in the refrigerator over night and the solid so formed was collected by filtration and was crystallized from appropriate solvent.

2a; lit. [18].

2b; Crystallization: EtOH; yield\%; 62\%; m.p.205-7 ${ }^{\circ} \mathrm{C}$; M. $\mathrm{FC}_{14} \mathrm{H}_{11} \mathrm{ClN}_{2} \mathrm{OS}$; M. Wt. 290.77; 'H-NMR.dppm ; 6.92-7.62 (m,7H,Ar-H), $3.72\left(\mathrm{~s}, 3 \mathrm{H}, \mathrm{OCH}_{3}\right), \quad 2.31(\mathrm{~s}, 1 \mathrm{H}$, exchangeable with $\left.\mathrm{D}_{2} \mathrm{O}, \mathrm{SH}\right) ;{ }^{13} \mathrm{c}$-NMR; $55.6\left(\mathrm{OCH}_{3}\right), 115.1,115.1,115.6,116.1,123.0$, 123.0, 124.4 (C-aromatic), $129.4\left(\mathrm{C}_{5}\right), 130.1(\mathrm{~N}-\mathrm{Ar}), 134.5\left(\mathrm{c}_{5}\right), 141.7\left(\mathrm{c}_{2}\right), 142.3\left(\mathrm{C}_{5}\right)$, $159.2\left(\mathrm{C}-\mathrm{OCH}_{3}\right)$; and MS (z/e\%) 291, $292(32,45), 185(100 \%)$.

2c; Crystallization: EtOH; yield\% 33; m.p.248-250 ${ }^{\circ} \mathrm{C}$; M. F. .C ${ }_{14} \mathrm{H}_{10} \mathrm{ClN}_{3} \mathrm{O}_{2} \mathrm{~S}$; M. Wt.. 319.77; 'H-NMR.dppm ; 7.34-8.25 (m,6H,Ar-H), 1.12 (s, 3H, $\left.\mathrm{CH}_{3}\right), 2.12(\mathrm{~s}, 1 \mathrm{H}$, exchangeable with $\mathrm{D}_{2} \mathrm{O}, \mathrm{SH}$ and $332,333(56,59), 199(100 \%)$.

3; Crystallization: EtOH; yield\% 70\%; m.p. 192-4 ${ }^{\circ} \mathrm{C}$; M. F. . $\mathrm{C}_{7} \mathrm{H}_{6} \mathrm{ClN}_{3} \mathrm{~S}$; M. Wt.. 199.67; IR, $\mathrm{cm}^{-1}$ : 3480- 3365, 'H-NMR.dppm; 6.82-7.42 (m,3H,Ar-H), 4.05 (s,2H, exchangeable with $\left.\mathrm{D}_{2} \mathrm{O}, \mathrm{NH}_{2}\right), 2.19\left(\mathrm{~s}, 1 \mathrm{H}\right.$, exchangeable with $\left.\mathrm{D}_{2} \mathrm{O}, \mathrm{SH}\right)$ and $\mathrm{MS},(\mathrm{z} / \mathrm{e} \%) 199,200$ $(35,39), 185(100 \%)$.

4a; Crystallization: $\mathrm{MeOH}$; yield\% $45 \%$; m.p. $228-30^{\circ} \mathrm{C}$; M. F. . $\mathrm{C}_{13} \mathrm{H}_{10} \mathrm{CIN}_{3} \mathrm{~S}$; M. Wt.. 275.76;

IR, $\quad \mathrm{cm}^{-1} .3180(\mathrm{NH}),{ }^{\prime} \mathrm{H}-\mathrm{NMR} . \mathrm{dppm} ; 8.62$ (s,b, exchangeable with D $\left.{ }_{2} \mathrm{O}, \mathrm{NH}\right), 7.42-$ $8.01(\mathrm{~m}, 8 \mathrm{H}, \mathrm{Ar}-\mathrm{H}), 2.09\left(\mathrm{~s}, 1 \mathrm{H}\right.$ exchangeable with $\left.\mathrm{D}_{2} \mathrm{O}, \mathrm{SH}\right)$ and $\mathrm{MS},(\mathrm{z} / \mathrm{e} \%) 276,277,278$ $(55,57,59), 134(100 \%)$.

4b; Crystallization: benzene; yield\% 45\%; m.p. $210-2{ }^{\circ} \mathrm{C}$; M. F. .C ${ }_{13} \mathrm{H}_{9} \mathrm{ClN}_{4} \mathrm{SO}_{2}$; M. Wt.. 320.76 
IR, $\quad \mathrm{cm}^{-1} ; 3160(\mathrm{NH}),{ }^{1} \mathrm{H}-\mathrm{NMR} . \mathrm{dppm} ; 8.12\left(\mathrm{~s}, \mathrm{~b}, 1 \mathrm{H}\right.$, exchangeable with $\left.\mathrm{D}_{2} \mathrm{O}, \mathrm{NH}\right), 7.12-$ 8.02 (m, 7H.Ar-H), 2.12 (s.! H, exchangeable with $\mathrm{D}_{2} \mathrm{O}, \mathrm{SH}$ ) and $\mathrm{MS}$. (z/e\%) 321,322 $(43,46), 134(100 \%)$.

2-(Arylamino)-5-choro-benzoxazle 5a-c

2-(hydrazino)-5-choro-benzoxazle 6

2-(Arylhydrazino)-5-choro-benzoxazle 7a,b

\section{General Procedure}

An equimolecular amounts $(0.37 \mathrm{~g}, 2 \mathrm{mmol})$ of 1 and appropriate aromatic amine (aniline, $p$-anisidine and $m$-nitro-o-toludine), hydrazine hydrate phenyl hydrazine or $p$-nitrophenyl hydrazine was stirred under refluxing temperature in $30 \mathrm{ml} \mathrm{n}$-butanol for $10 \mathrm{hrs}$. The reaction mixture was cooled was left in the refrigerator over night and the solid so formed was collected by filtration and was crystallized from appropriate solvent .

5a; Crystallization: EtOH; yield\% 62; m.p.231-3 ${ }^{\circ} \mathrm{C}$; M. F. . $\mathrm{C}_{13} \mathrm{H}_{9} \mathrm{ClN}_{2} \mathrm{O}$; M. Wt. 244.76; IR, $\mathrm{cm}^{-1} ; 3165$ (NH), ), ${ }^{1} \mathrm{H}-\mathrm{NMR} . d p p m$ 7.41-8.30 (m,8H,Ar-H), 7.82 (s,b, 1 H, exchangeable with $\mathrm{D}_{2} \mathrm{O}, \mathrm{NH}$ ) and MS. (z/e\%) 244,245 (45,49), 189(100\%).

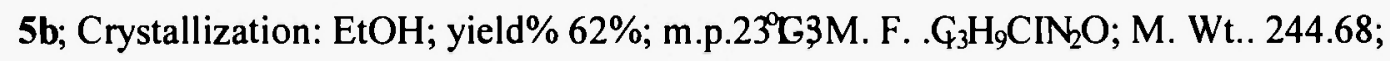

IR, $\quad \mathrm{cm}^{-1} ; 3167(\mathrm{NH}), 7.32-8.10(\mathrm{~m}, 7 \mathrm{H}, \mathrm{Ar}-\mathrm{H}), 6.02\left(\mathrm{~s}, \mathrm{~b}, 1 \mathrm{H}\right.$, exchangeable with $\mathrm{D} \quad{ }_{2} \mathrm{O}$, $\mathrm{NH}), 1.64\left(\mathrm{~s}, 3 \mathrm{H}, \mathrm{CH}_{3}\right)$ and MS. (z/e\%) 275,276(44,46), 195(10\%).

5c; Crystallization: benzene; yield\% 32\%; m.p.26G-3M. F. $\mathrm{G}_{4} \mathrm{H}_{10} \mathrm{ClN}_{3} \mathrm{O}_{3}$; M. Wt.. 303.70; IR, $\mathrm{cm}^{-1} ; 3210(\mathrm{NH}),{ }^{1} \mathrm{H}-\mathrm{NMR} . d p p m$ 7.30-8.08 (m,6H,Ar-H), 6.1212 (s,b, 1 H, exchangeable with $\left.\mathrm{D}_{2} \mathrm{O}, \mathrm{NH}\right), 1.12(\mathrm{~s}, 3 \mathrm{H}, \mathrm{CH})$ and $\mathrm{MS}$. (z/e\%) 320, $321(52,54), 189(100 \%)$.

6; Crystallization: EtOH; yield\% 75; m.p.235-7 ${ }^{\circ} \mathrm{C}$; M. F. . $\mathrm{C}_{7} \mathrm{H}_{6} \mathrm{ClN}_{3} \mathrm{O}$; M. Wt.. 183.56; IR, $\quad \mathrm{cm}^{-1} ; 3467-3350$ and $3190\left(\mathrm{NH}_{2}, \mathrm{NH}\right) ;{ }^{1} \mathrm{H}$-NMR.dppm $8.52(\mathrm{~s}, \mathrm{~b}, 1 \mathrm{H}$, exchangeable with $\left.\mathrm{D}_{2} \mathrm{O}, \mathrm{NH}\right), 8.01\left(\mathrm{~s}, 2 \mathrm{H}\right.$, exchangeable with $\left.\mathrm{D}{ }_{2} \mathrm{O}, \mathrm{NH}_{2}\right), 6.82-7.43(\mathrm{~m}, 3 \mathrm{H}, \mathrm{Ar}-\mathrm{H})$ and MS. (z/e\%) 183 (56), 185 (100\%).

7a; Crystallization: ChCN; yield\% 46; m.p.265ํㅜㅇ M. F. $\mathrm{G}_{3} \mathrm{H}_{10} \mathrm{CIN}_{3} \mathrm{O}$; M. Wt.. 259.69; IR, $\mathrm{cm}^{-1} 3210,3120$ (2NH) ), ${ }^{1} \mathrm{H}$-NMR.dppm; 7.43-8.12 (m,8H,Ar-H), 7.30, 6.24 (s,b,eachlH, exchangeable with $\left.\mathrm{D}_{2} \mathrm{O}, \mathrm{NH}\right)$ and MS. (z/e\%) 259, $260(25,27), 189(100 \%)$. 7b; Crystallization: n-butanol; yield\% 55; m.p.240;2M. F. . $\mathrm{G}_{3} \mathrm{H}_{9} \mathrm{ClN}_{4} \mathrm{O}_{3}$; M. Wt.. 304.69; 
IR, $\mathrm{cm}^{-1} 3110,3120(2 \mathrm{NH})$ ), 'H-NMR.dppm; 8.72,7.51 (s,b.each IH, exchangeable with $\left.\mathrm{D}_{2} \mathrm{O}, \mathrm{NH}\right), 7,31-7.81(\mathrm{~m}, 7 \mathrm{H}, \mathrm{Ar}-\mathrm{H})$ and MS. (z/e\%) 305, $306(43,49), 189(100 \%)$.

\section{7-Chloro-2-methyl-benzo[4,5]imidazo[2,1b][1,2,4]thiadiazole 8}

A solution of $3(0.99 \mathrm{~g}, 5 \mathrm{mmol})$ in $10 \mathrm{ml}$ of triethyl orthoacetate was heated under reflux for 10 hrs. The excess triethyl orthoacetate was evaporated under vacuum to obtain wax material. It was solidified from methanol and the obtained solid was crystallized from n-butanol . 8; Crystallization: n-butanol; yield\% 45; m.p.256-8 ${ }^{\circ} \mathrm{C}$; M. F. $\mathrm{C}_{9} \mathrm{H}_{6} \mathrm{ClN}_{3} \mathrm{~S}$; M. Wt. 223.69; C 'H-NMR.dppm; 7.45-8.21 (m,3H,Ar-H), 2.16 (s,3H, $\left.\mathrm{CH}_{3}\right)$ and MS. (z/e\%) 224,225 $(54,56), 219(100 \%)$.

\section{7-Chloro-2-oxo-benzo[4,5]imidazo[2,1 b][1,2,4]thiadiazole 9}

To a solution of $3(0.99 \mathrm{~g}, 5 \mathrm{mmol})$ in $10 \mathrm{ml}$ pyridine, ethyl chloroformate $(0.56 \mathrm{~g}, 5 \mathrm{mmol})$ was added in drop wise. The reaction mixture was heated under reflux for $12 \mathrm{hrs}$. The reaction mixture was cooled and was poured into crushed ice, the solid formed was collected by filtration and was crystallized from benzene.

9; Crystallization: benzene; yield\% 58; m.p.244-6 ${ }^{\circ} \mathrm{C}$; M. F. . $\mathrm{C}_{8} \mathrm{H}_{4} \mathrm{ClN}_{3} \mathrm{O}$; M. Wt.. 225.66;

IR $\mathrm{cm}^{-1} 3120(\mathrm{NH})$ and 1670 (cyclic amide), 'H-NMR.dppm; 7.52-7.98 (m,3H,Ar-H), 5.02 (s,b, exchangeable with $\left.\mathrm{D}_{2} \mathrm{O}, \mathrm{OH}\right)$ and $\mathrm{MS}$. (z/e\%)225.59 (53\%), 55 (100).

\section{7-Chloro-2-aryl-benzo[4,5]imidazo[2,1b][1,2,4]thiadiazole 10a-c}

An equimolecular amounts of $3(0.99 \mathrm{~g}, 5 \mathrm{mmol})$ and appropriate aromatic aldehyde (panisaldehyde, $p$-nitrobenzaldehyde and $\mathrm{N}, \mathrm{N}$-dimethlaminobenzaldehyde in $25 \mathrm{ml}$ of dioxane in presence of catalytic amounts of pipridine was heated for $9 \mathrm{hrs}$. The reaction mixture was cooled, the solid so formed was collected by filtration and was crystallized form appropriate solvent .

10a; Crystallization: n-butanol; yield\% 42;m.p.236-8 C;M.F. $\mathrm{C}_{15} \mathrm{H}_{10} \mathrm{CIN}_{3} \mathrm{SO}$; M.Wt. 315.79; ${ }^{\prime} \mathrm{H}-N M R . d p p m ; 7.34-8.10$ (m,7H,Ar-H), 1.93 (s, 3H,OCH); ${ }^{13} \mathrm{C}-\mathrm{NMR} ; 55.9\left(\mathrm{OCH}_{3}\right), 115.4$, $116.7,124.3,127.8,127.8,129.5,129.5,132.1$ (C-aromatic), $125.2\left(\mathbb{C} 129.1\left(\mathrm{C}_{7}\right), 142.1\left(\mathrm{C}_{5}\right)\right.$, $142.5\left(\mathrm{C}_{2}\right), 140.2,172.0(\mathrm{~N}-\mathrm{C}-\mathrm{S})$ and MS. (z/e\% ) 315,316 (24, 25), $134(100 \%)$. 
10b; Crystallization: EtOH; yield\% 48; m.p.251- $=$; ; M. F. . $\mathrm{G}_{4} \mathrm{H}_{7} \mathrm{ClN}_{4} \mathrm{SO}_{2}$; M. Wt.. 330.76;

${ }^{1}$ H-NMR. dppm; 8.85-7.34 (m,7H,Ar-H) and MS. (z/e\%) 331,332 (32,36), 123 (100\%).

10c; Crystallization: EtOH; yield\% 65; m.p.216-8 ${ }^{\circ} \mathrm{C}$; M. F. . $\mathrm{C}_{16} \mathrm{H}_{13} \mathrm{ClN}_{4} \mathrm{~S}$; M. Wt..

328.83;. ' 'H-NMR.dppm; 7.01-7.89 (m,7H,Ar-H),3.91 (s,6H,N(CH$\left.)_{2}\right)$, and MS. (z/e\%)

$328,329(37,39 \%), 134(100 \%)$.

\section{References:}

1. Yalçm Yalçin, E.Sener, T.Õzden, S.Õzden, and A. Akmin; Eur. J. Med. Chem 25, 705-8 (1990).

2. I. Yalçm Yalçin, I. Õren, E. Sener, A. Akin. and N. Uøałv,tEur. J. Med. Chem27,401-406 (1992).

3. I.Yalçmin, and E. Senełnt. J. Rharm.98,1-9 (1993).

4. E. Sener, I. Yalçmin, and E. Sungpusant. Struct. Act. Reld10,223-228 (1991).

5. E. Sener. I. Yalçm Yalçin, Õ. Temiz, I. Õren. A. Aland,N. UcavturkUcarturłF,armaco52, 99-103 (1997).

6. I.Õren, O. Temiz, I. Yalçm, E. Sener, and A. Akirnģeim. Forsch./Drug ret7,1393-1397 (1997).

7. Õ. Temiz, I.Õren, E. Sener, I. Yalçm, and N. Ucarfuntugaco.53,337- 341 (1998.)

8. I.Õren, Õ. Temiz, I. Yalçm, E. Sener, and N. Altałlar;,J. Pharm. Sc7., 153-160 (1998).

9. V. A. Zubkov, S. N. Kovalenko, V. P. Chernykh, and S. M. IvlKohim. Geterotsikl. Soedin 760-766 (1994).

10. W. LwowskiSynthesis71(1971).

11. S. N. Kovalenko, V. A. Zubkov, V. P. Chenykh, A. V. Turov, and S. M. Ivkov; Khim. Geterotsikl. Soedin.86-192 (1996).

12. A.S. Aly, N. M. Fathy, S. A. Samira, Swelam and F. M. E. Abdel-Magiqd; J. Pharm. Sci. 36(177), 1-7 (1995)

13. S. A. SwelamLndian J. Heterocyclic Chemistrfi; 147 (1998).

14. M. E. A. Zaki, N. M. Fawzy, and S. A. SweldraleculesOnline3,1 (1999).

15. S. A. Swelam, O. I. Abd-El-Salam, and M. E. A. ZaWerb. Chem. So64(11), 655 (1999).

16. A. Harizi, A. Romdhane, and Z. Mightrahedronetters41(31)5833-5835 (2000).

17. S. A. Swelam, O. A. Fathalla, M. .E. A.. Zaki and H.F.AlGgypt. J. Chem.47(6),677-692 (2004).

18. http://www.google.com/patents?hl=en\&lr=\&vid=USPAT4814329\&id= 6VVvAAAAEBAJ\&oi=fnd\&da =Yakugaku+Zasshi $+(1958) .78+1378-82$

Received on August 15, 2007 